\title{
Congenital Hypothyroidism: A Report of Two Isolated Cases at National Hospital Abuja
}

\author{
N. Y. Papka, I. B. Babaniyi and N. Hamza \\ Department of Pediatrics, National Hospital Abuja, Federal Capital Territory, 900211, Nigeria
}

\begin{abstract}
Background: Congenital hypothyroidism is a disorder of thyroid gland morphogenesis. It is the commonest endocrine disorder in newborns occurring in 1:4,000 to 1:3,000 live births. The incidence in Nigeria has not been well documented probably due to lack of neonatal screening. Aim/objectives: To raise awareness of physicians to have a high index of suspicion for this treatable cause of mental retardation in children presenting with unusual symptoms. Case presentation: Case 1 presented to our hospital at 18 months of age with a respiratory tract infection. She had delayed developmental milestones and coarse facies among other features. This prompted a work up for hypothyroidism. Thyroid function tests done showed a profoundly hypothyroid picture and she was commenced on levothyroxine. Case 2 was referred to our facility at ninth week of age on suspicion of a congenital heart disease. The parents had complained of dry skin to the referring physician. She had a hypothyroid profile on laboratory evaluation. Conclusion: Congenital hypothyroidism should be suspected in children with unusual symptoms as it is a treatable cause of short stature and mental retardation. The introduction of newborn screening will go a long way in identifying the children in need of urgent thyroid replacement to prevent the negative consequences of untreated hypothyroidism.
\end{abstract}

Key words: Congenital hypothyroidism, neonatal screening, mental retardation.

\section{Introduction}

Congenital hypothyroidism is a common endocrine disorder with a prevalence of 1:4,000 to 1:3,000 [1, 2]. It has long been recognized as a disorder that requires neonatal screening due to its ability to cause long-term neuropsychological and motor abnormalities [2, 3]. Newborn screening and thyroid therapy started within 2 weeks of age can normalize cognitive development [2]. Unfortunately newborn screening is almost nonexistent in low and middle income countries and Nigeria is one of them [4, 5]. We present these 2 cases to raise awareness of physicians to have a high index of suspicion for this treatable cause of mental retardation in children presenting with unusual symptoms.

\section{Case Analysis}

\subsection{Case 1}

AI presented at eighteen months of age with

Corresponding author: Dr N. Y. Papka, MB BS, FMCPaed, Consultant Pediatrician/Pediatric Cardiologist, research field: pediatrics. Email: nubwayusufu@yahoo.com. complaints of vomiting and coughs of three days duration. There were no loose stools or fever. Past medical history was significant for weakness and poor feeding that led to child being force-fed and at six months of age she choked and had multiple convulsions with loss of consciousness. She was managed for anoxic brain injury from severe aspiration pneumonitis. She was delivered following a supervised pregnancy with no antenatal illnesses nor use of unprescribed medications including herbal concoctions. Baby was delivered at term; birth weight unknown with no neonatal problems. She was up to date with immunization. Motor milestones were delayed as she achieved neck control at five months and crawled at one year. She had just learnt to stand with support a few days prior to current illness. She is the third child of parents. First is a six-year-old male; alive and well. The second was a male that died in neonatal period due to complications of prematurity. Both parents have some formal education and are gainfully employed.

Examination revealed a well grown hypoactive child 
with coarse skin. She was febrile, mildly pale, with obvious macrocephaly, low set ears and flat nasal bridge (see Fig. 1). She was stunted but weight was at 50th centile and occipito-frontal circumference was above the 95th centile for age and sex. The heart rate was one hundred and fourteen beats per minute with a blood pressure of 76/45 mmHg. The heart sounds were normal and the chest was clear. She was hypotonic and had a reducible umbilical hernia. There was an anterior neck swelling that measured $6 \mathrm{~cm} \times 4 \mathrm{~cm}$ and enlarged hyperaemic tonsils. A diagnosis of pharyngotosilitis with hypothyroidism was entertained. She had a profoundly hypothyroid profile with T3 level $<0.19$ $\mathrm{ng} / \mathrm{mL}$ (normal 0.92-2.48 ng/mL), T4 of $4.83 \mathrm{ng} / \mathrm{mL}$ (normal 59.5-146.9 $\mathrm{ng} / \mathrm{mL}$ ) and a thyrotropin level of $74.4 \mathrm{uIU} / \mathrm{mL}$ (normal 0.7-5.97 uIU/mL). The free T3 was $1.83 \mathrm{pmol} / \mathrm{L}$ (normal 3.1-6.8 $\mathrm{pmol} / \mathrm{L}$ ) and the free T4 was 1.44 (normal 12-22 pmol/L). The neck ultrasound showed an enlarged thyroid gland. She was commenced on levothyroxine and has since been on follow up. She has shown some improvement in linear growth and mild improvement in cognitive functioning despite the delay in diagnosis and treatment. At 3 years she started nursery and could recite some alphabets and count 1 to 5 correctly.

\subsection{Case 2}

GL presented at age of 9 weeks on referral for suspected heart disease. The parents had complained of dry skin, a left groin swelling and decreased activity compared to their other children. The pregnancy was supervised from four months and essentially uneventful. The delivery was at term in a car while on transit to a hospital. She cried well at birth, but the birth weight was not known. There were no neonatal problems. She is up-to-date with immunizations and has just achieved social smile. The child is the fifth of parents, older siblings are alive and well. Both parents have secondary level of education. Father is a commercial motorcyclist while mother is a petty trader.

Examination revealed a hypoactive child with coarse facies, hoarse cry, puffy eye lids, low set ears, hypertelorism, flat nasal bridge and low hair line (see Fig. 2). The tongue was protruding and she turned dusky when crying. The skin was mottled and she had no pedal oedema. The lung fields were clear with normal first and second heart sounds and a grade 2/6 ejection systolic murmur at the left upper sternal border. There was also a reducible umbilical and left inguinal hernia with both anterior and posterior fontanelles open. The occipito-frontal circumference was $37 \mathrm{~cm}$ and the tone was normal. A diagnosis of congenital hypothyroidism with a possible congenital heart disease was entertained and tests were ordered. The serum T3 was $<0.195 \mathrm{ng} / \mathrm{mL}$, T4 was $4.41 \mathrm{ng} / \mathrm{mL}$ and thyrotropin was $>100 \mathrm{uIU} / \mathrm{mL}$. The echocardiography revealed mild stenosis of the left pulmonary artery and mild tricuspid regurgitation. The thyroid ultrasound scan

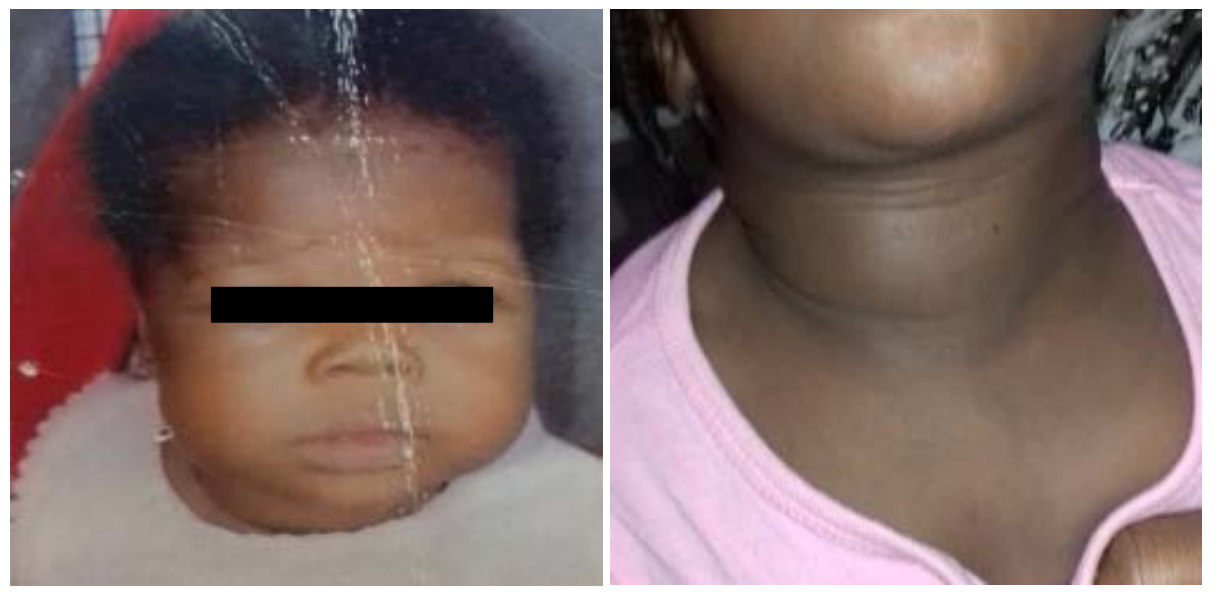

Fig. 1 Right: Case 1 in infancy, with typical coarse hypothyroid facies and low hair line. Left: same patient with anterior neck swelling. 


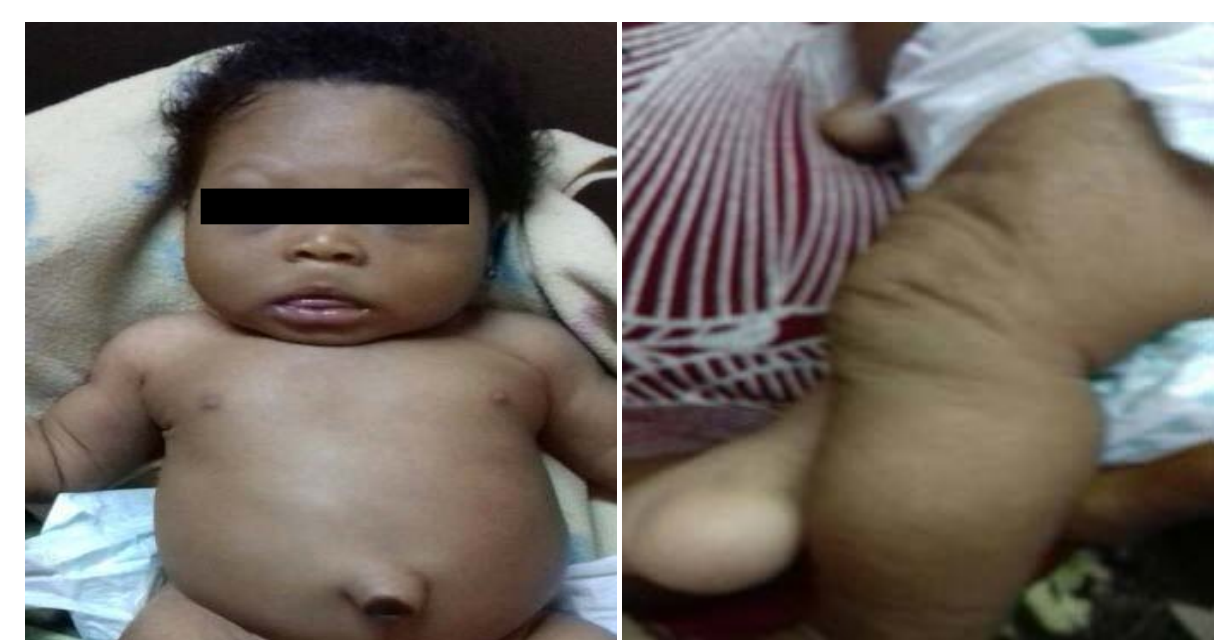

Fig. 2 Right: Case 2 with large protruding tongue, coarse facies, widely spaced nipples and umbilical hernia. Left: Same child with dry mottled skin.

revealed a normal sized thyroid gland. She was commenced on L-thyroxin two weeks later as parents had to source for the funds. She was subsequently seen twice after commencement of L-thyroxine and examination revealed reduction in the hoarseness of voice and puffy eyelids. The parents were unable to keep up with the cost of follow up investigations and medications and hence the patient was lost to follow up from fifteen weeks of age.

\section{Discussion}

Congenital hypothyroidism is the commonest neonatal endocrine disorder and if untreated it results in irreversible neuropsychological deficits [1, 2, 4].

The local prevalence in Nigeria is not well documented probably due to lack of new born screening $[3,5,6]$. The prevalence is lowest in blacks and highest in the Hispanics and Asians [1]. It is sporadic though up to $2 \%$ of dysgenesis type are familial [7]. It can be transient or permanent with the former being as a result of maternally transmitted anti-thyroid antibodies [7]. As the name implies, the thyroid function in transient type normalizes within few weeks to months [7]. The permanent type results from an absent or ectopic gland (most common), dyshormonogenesis in normally situated thyroid gland or defects from thyrotropin receptors. It appears both of our patients may have the dyshormonogenesis type [7].
Concentrations of thyroid hormone are low in the fetus during the first half of pregnancy [2]. During this time, the fetus is entirely dependent on maternal transplacental transfer of this enzyme which is required for maturation of multiple organ systems especially the brain [2]. After delivery, the fetus is entirely dependent on its own production of thyroid hormone and maternally transferred hormone falls within the first 48 to 72 hours of life [1, 2, 8]. As such, most children with congenital hypothyroidism appear normal at birth though pregnancy may have been prolonged for up to forty two weeks [7]. An umbilical hernia is present in up to $32 \%$ as seen in our patients [1]. These children are usually quiet and may sleep through the night [1]. Prolonged neonatal jaundice is one of the common presentations but was not depicted in any of our patients [1]. If untreated, by three months of age most infants will have the full blown hypothyroid picture as was present in the second case $[1,2,9]$. These include feeding difficulties, protruding large tongue, coarse facies, hoarse voice, dry mottled skin, pseudo-hypertelorism, wide posterior fontanelle, sutural diastasis and hypotonia among others [1, 2, 9]. The feeding difficulty in our first case resulted in the child being force-fed which led to severe aspiration pneumonitis and hypoxic brain injury. Up to $7 \%$ of non-thyroidal congenital anomaly rate has been reported in children with congenital hypothyroidism, 
as seen in case 2 who had mild left pulmonary artery stenosis [7].

By the age of three months, significant irreversible mental retardation would have occurred in untreated children [9]. Lack of a high index of suspicion may explain why AI was missed even at her first hospital admission at six months of age.

L-thyroxine when started early in children detected by newborn screening, results in an excellent cognitive outcome [2]. However delaying treatment beyond two months of age leaves the child with residual cognitive deficits even though the stature and physical appearance may normalise. AI had some improvement in skeletal growth, overall appearance and some neurocognitive development despite the very late age at diagnosis and commencement of therapy. GL was diagnosed much earlier than AI though still not at an optimal age but the financial status of the family and cost of follow up investigations and medications has denied her the benefit of improved neurocognitive development.

\section{Conclusions}

Congenital hypothyroidism is the commonest paediatric endocrine disorder globally and may be in Nigeria if neonatal screening is introduced. In the absence of neonatal screening, a high index of suspicion may allow early identification of these infants with prompt treatment to prevent its dreaded complication of mental retardation.

\section{References}

[1] Rastogi, M. V., and Lafranchi, S. H. 2010. "Congenital Hypothyroidism Definition and Classification.” Orphanet $J$ Rare Dis.

[2] American Academy of Pediatrics, Rose, S. R., Section on Endocrinology and Committee on Genetics, American Thyroid Association, Brown, R. S., Public Health Committee, et al. 2006. "Update of Newborn Screening and Therapy for Congenital Hypothyroidism.” Pediatrics 117: 2290-304.

[3] Oyenusi, E., Ajayi, E., Akeredolu, F., et al. 2017. "Pattern of Thyroid Disorders in Children and Adolescents Seen at the Lagos University Teaching Hospital, Nigeria, over a 10-Year Period.” Niger Med J 58: 101.

[4] Hamdoun, E., Karachunski, P., Nathan, B., et al. 2016. "Case Report: The Specter of Untreated Congenital Hypothyroidism in Immigrant Families.” Pediatrics 137: e20153418.

[5] Adeniran, K. A., Okolo, A. A., and Onyiriuka, A. N. "Thyroid Profile of Term Appropriate for Gestational Age Neonates in Nigeria: A Forerunner to Screening for Congenital Hypothyroidism.”

[6] Kayode-Adedeji, B., and Alikah, S. 2012. International Journal of Basic, Applied and Innovative Research IJBAIR. Anthonio Research Centerhttps://www.ajol.info/index.php/ijbair/article/view/ 121886 (accessed 18 July 2018).

[7] Park, S. M., and Chatterjee, K. 2005. "Genetics of Congenital Hypothyroidism.” J Med Genet 42: 379-89.

[8] Léger, J., Olivieri, A., Donaldson, M., et al. 2014. "European Society for Paediatric Endocrinology Consensus Guidelines on Screening, Diagnosis, and Management of Congenital Hypothyroidism.” Horm Res Paediatr 81: 80-103.

[9] Dubuist, J., Glorieux, J., Richer, F., et al. 1996. “Outcome of Severe Congenital Hypothyroidism: Closing the Developmental Gap with Early High Dose Levothyroxine Treatment.” J Clin Endocrinol Metab Copyr Endocr Soc. 